\title{
Lateinamerikanische Verfassungen: Zwischen Autokratismus und Demokratisierung
}

\author{
Von Marcelo Neves
}

\section{I.}

Beobachtet man die Verfassungsentwicklung Lateinamerikas in den letzten dreißig Jahren, so läßt sich zwar feststellen, daß sich trotz einiger Ausnahmen die Demokratisierung als eine allgemeine Tendenz durchgesetzt hat. Im Gegensatz zum Vorherrschen der Autokratie in den sechziger und siebziger Jahren haben sich in den achtziger Jahren konstitutionalisierende Trends aufgetan, die sich in den neunziger Jahren zu stabilisieren scheinen. Aber es ist hier angebracht, die Frage aufzuwerfen, in welchem Maße die erneuerten politischen Strukturen wirklich konsolidiert sind und die entsprechenden Verfassungstexte als normative Modelle der Erwartungsorientierung und Verhaltenssteuerung konkretisiert bzw. realisiert werden. Das zu behandelnde Problem liegt genau darin, daß besonders im Fall der politischen Entwicklung Lateinamerikas der Aufbau und die Verwirklichung des demokratischen Rechtsstaats nicht einfach durch die Überwindung der Autokratie garantiert werden. Vielmehr ist festzustellen, daß die unzureichende Realisierung von demokratischen Verfassungsmustern einen der wichtigsten Faktoren für das Überbleibsel des autokratischen Diskurses als einer relevanten Variable im Rahmen der Machtverhältnisse in den lateinamerikanischen Ländern bildet. Unter diesen Umständen stehen Autokratismus und Demokratisierung nicht wie herkömmlicherweise im widersprüchlichen Verhältnis, sondern in einer spannungsreichen und paradoxen Beziehung von gleichzeitiger Divergenz und Ergänzung zueinander. Freilich geht die Problematik der mangelhaften Konkretisierung demokratischrechtsstaatlicher Verfassungstexte weit über die Frage der Gefahr bzw. des Wiederauflebens autokratischer Erfahrungen hinaus. Sie führt umfassender auf zugrundeliegende, geschichtlich bedingte und festgefügte soziale Strukturen und Beziehungen zurück, die als Hindernisse für die Ausdifferenzierung des Rechts und die Konstruktion einer pluralistischen Öffentlichkeit wirken.

Im folgenden geht es vornehmlich nicht um eine eingehende vergleichende Analyse der unterschiedlichen Verfassungen Lateinamerikas, was den Rahmen dieses Symposiums sprengen würde. Vielmehr soll versucht werden, einige allgemeine Züge der Verfassungsentwicklung Lateinamerikas zu erörtern, besonders im Hinblick auf den Übergang von Autoritarismus zur Demokratisierung in den letzten drei Jahrzehnten. Damit soll allerdings die Mannigfaltigkeit der politischen und rechtlichen Wirklichkeit Lateinamerikas nicht verkannt werden. Ich erhebe selbstverständlich keinen Anspruch auf eine vollkommene 
Darstellung. So ist hier z. B. auf die politische Entwicklung Mexikos hinzuweisen, wo sich seit Inkrafttreten des sozialdemokratischen Verfassungstextes von 1917 eine stabile Erfahrung von Verfassungsnominalismus durchsetzte und somit in den letzten Jahren nicht vom Übergang vom Autokratismus zur Demokratisierung die Rede sein kann. Auf andere Weise zeigt sich in Peru eine autoritäre Gegentendenz zum vorherrschenden Demokratisierungstrend. Außerdem handelt es sich hier nicht um eine fatalistische Diagnostik, als ob keine Möglichkeit der Überwindung der problematischen Situation bestehen würde und keine Alternative dazu auftreten könnte, sondern um eine historisch bedingte Interpretation von dominierenden Entwicklungstendenzen.

In Anbetracht der geschichtlich dominierenden Pendelbeziehung zwischen Verfassungsinstrumentalismus als Ausdruck von Autokratie und Verfassungssymbolismus als Ausdruck von Demokratisierung wird zunächst die lateinamerikanische Autokratie als autoritäres Regime charakterisiert und die in den sechziger und siebziger Jahren auftauchenden Militärregimes erörtert (II). Danach werde ich das Problem der unzureichenden Konkretisierung demokratischer Verfassungstexte behandeln; anschließend soll die überwiegend symbolische Funktion rechtsstaatlicher Verfassungsurkunden berücksichtigt werden (III). Ferner möchte ich darauf hinweisen, daß zwischen Autokratismus und demokratischem Verfassungssymbolismus, also jedenfalls mangels normativer Verfassung, die Grenzen von Politik und Recht undurchsichtig werden, vor allem zu Lasten der Autonomie des Rechtssystems, nämlich durch dessen Unterordnung unter die Politik; im Anschluß daran sollen die Hindernisse für die Konstruktion einer pluralistischen Öffentlichkeit und folglich für den Aufbau und die Verwirklichung des Rechtsstaates in Betracht kommen (IV). Zum Schluß wird hervorgehoben, daß die Problemlösung nicht einfach auf die Ebene der Verfassunggebung bzw. Verfassungsänderung reduziert werden kann und daß sie von einem umfassenden Abbau von Hindernissen der Verfassungskonkretisierung abhängt; hinzu kommt eine kritische Bemerkung über die verfassungsreformistische Rhetorik, besonders im Hinblick auf den gegenwärtigen Verfassungsdiskurs für die Entkonstitutionalisierung in Brasilien (V).

\section{II.}

Es läßt sich feststellen, daß sich im Verlauf der lateinamerikanischen Verfassungsgeschichte eine Pendelbeziehung zwischen Autokratismus und Demokratisierung in der Form jeweils instrumentalistischer und symbolischer Verfassungen als typisch herauskristallisiert hat. In der politischen Rhetorik drückt sich dies durch die Handhabung der einander ersetzenden Formeln "Wiederherstellung der Ordnung" und "Wiederherstellung der Demokratie" aus. Besonders unter den autoritär orientierten Publizisten wird diese Situation oft als Spannung bzw. Konflikt zwischen Verfassungsrealismus und Verfassungsidealismus vor- 
gestellt. ${ }^{1}$ In beiden Richtungen aber läuft das Problem einerseits auf mangelhafte Autonomie des Rechts bzw. des Staates gegenüber diffusen, sich durch Herrschaftsprivilegien und Exklusion, gute Beziehungen und Klientenverhältnisse charakterisierten Gesellschaftsstrukturen im Inland und Ausland, andererseits und damit zusammenhängend auf das Nichtvorhandensein einer den Staat legitimierenden, umfassenden, pluralistischen Öffentlichkeit hinaus. Also sind auch im Fall der autokratischen Erfahrungen die Stärke des Staats und dessen Verbindung mit den entsprechenden nationalen Wirklichkeiten bzw. Wurzeln nur scheinbare. Vielmehr ist die direkte Unterordnung des Staates unter politische Partikularismen und private Interessen festzustellen.

Unterscheidet man mit Loewenstein im Rahmen des Grundbegriffs der Autokratie idealtypisch das sich "auf die gesamte politische, gesellschaftliche und moralische Ordnung der Staatsdynamik" beziehende Konzept des Totalitarismus und den sich "mehr auf die Regierungsstruktur" beziehenden Begriff des Autoritarismus, ${ }^{2}$ zeigt sich sehr deutlich, daß sich die lateinamerikanischen Autokratien als autoritäre Regimes charakterisieren lassen. Dabei entwickelte sich keine Ideologie mit Anspruch auf unmittelbare Gültigkeit in allen Bereichen der Gesellschaft. Es gibt also keine gesamtgesellschaftliche Entdifferenzierung durch ein in der Spitze stehendes politisches System wie tendenziell im Totalitarismus. Es fehlt auch an ideologischer Konsistenz, so daß es nicht angebracht ist, nach einer früheren, auf einen anderen Kontext gerichteten Formulierung Luhmanns ${ }^{3}$ zu behaupten, es gehe um "ideologische integrierte Systeme" im Gegensatz zu den Rechtsstaaten. In den autoritären Regimes Lateinamerikas ist die Regierung oder umfassender der Staat an verschiedenste politische Konstellationen gebunden und mit unterschiedlichsten sozialen Gebilden verflochten, die weder zu einer Legitimation durch Verfahren noch zur Identifikation des Individuums oder der Gesellschaft mit dem Staat beitragen, sondern als diffuse und partikulare Mechanismen der Unterstützung ${ }^{4}$ dienen. Daraus ergibt sich ein Durcheinander von

Vgl. Vianna, Oliveira, O Idealismo da Constituição, 2. Aufl., São Paulo / Rio de Janeiro / Porto Alegre 1939, insbes. S. 7 ff. u. S. 303 ff.; Reale, Miguel, Momentos decisivos do constitucionalismo brasileiro, in: Revista de Informação Legislativa (Brasília), Jg. 20, N. 77, 1983, S. 57-68; Torres, Alberto, A organização nacional: Primeira parte, A Constituição, 3. Aufl., São Paulo 1978 (1. Aufl. 1914), S. $160 \mathrm{ff}$.

2 Loewenstein, Karl, Verfassungslehre, dt. Übers. v. Rüdiger Boerner, 3. Aufl., Tübingen 1975, S. 53, 55. "In der Regel begnügt sich das autoritäre Regime mit der politischen Kontrolle des Staates, ohne Anspruch darauf zu erheben, das gesamte sozio-ökonomische Leben der Gemeinschaft zu beherrschen oder ihre geistige Haltung nach seinem Ebenbild zu formen" (ibid., S. 53).

Luhmann, Niklas, Positives Recht und Ideologie, in: ders., Soziologische Aufklärung 1: Aufsätze zur Theorie sozialer Systeme, 5. Aufl., Opladen 1984, S. 178-203, S. $193 \mathrm{ff}$. 
Einflüssen, das sich auf der Ebene der Verfassungscharta und der Ausnahmegesetze als Inkonsistenz und "kasuistische" Lösung widerspiegelt. ${ }^{5}$

In den Militärregimes der sechziger und siebziger Jahren läßt sich die Vermischung nationalistischer Rhetorik, die auch in die Normtextgebung hineingreift, mit der Unterordnung der realen Politik unter die strategischen Interessen der in der Region stärksten Großmacht, d.h. der Vereinigten Staaten ${ }^{6}$, und der multinationalen Konzerne beobachten. Außerdem liegt unter einer auf der staatlichen Bühne stehenden Politik der wirtschaftlichen Effizienz eine politische Praxis von Subsidien für ökonomisch privilegierten Gruppen, die es nicht ertragen, die Risiken der Marktwirtschaft einzugehen. Gleichzeitig aber erfordern die exkludierenden Gesellschaftsstrukturen und -beziehungen im Zusammenhang mit dem Fehlen von wirksamer bzw. ernstzunehmender sozialstaatlicher Politik Klientenverhältnisse nicht nur mit den privilegierten Gruppen, sondern auch mit den Unterschichten. Unter direktem Druck von Innen und Außen, von "Oben" und "Unten" entstanden und verharrten die Militärregimes also ohne genügende Identität, um einen starken Staat zu konstruieren. Im Gegenteil kann man auch im Hinblick auf die in den sechziger und siebziger Jahren errichteten autoritären Militärregimes feststellen, daß sich die Grenzen zwischen dem Staatlichen und dem Privaten nicht durchzusetzen vermochten. Aber es handelt sich hier nicht um traditionellen, auf moralische Prinzipien zurückgehenden Patrimonialismus, sondern um diffuse, besonders von konkreten Interessen der Privilegierten und doch auch von unmittelbaren Bedürfnissen der »Marginalisierten« abhängige Privatisierung des Staatsapparates.

Auf anderer Ebene, derjenigen der Verhältnisse zwischen Politik und Recht, drückt sich selbstverständlich eine Hypertrophie der Staatsherrschaft zu Lasten der Menschenrechte aus. Dies läßt sich als direkte Überordnung der Politik über das Recht interpretieren. Sie ist "direkt" in dem Sinne, daß die Grundrechte, die Gewaltenteilung und die freie, gleiche und allgemeine Wahl als wichtigste Institutionen des demokratischen Rechtsstaats unmittelbar im Rahmen der Rechtssetzung betroffen werden. Das schließt zwar nicht aus, daß in den Verfassungsurkunden der autoritären Regimes die Erklärung der Grundrechte, das Wahlverfahren und das Prinzip der Gewaltenteilung vorgesehen sind. Aber in Anbetracht anderer Verfassungsvorschriften sowie von Ausnahmegesetzen mit verfassungsrechtlicher Kraft werden diese rechtsstaatlichen Institutionen in ihrer Bedeutung so begrenzt, daß man von ihrer manifesten Entstellung durch Grundgesetzgebung sprechen kann. Außerdem wird

So kennzeichnete Loewenstein, Karl, Brazil under Vargas, New York 1942, S. 122, ironisch die brasilianische Verfassungscharta von 1937 als "internationales Tuttifrutti" und "konstitutionellen Cocktail".

6

Vgl. am Beispiel des Militärputsches von 1964 in Brasilien mit vielen Belegen Sá Corrêa, Marcos, 1964 visto pela Casa Branca, Porto Alegre 1977; umfassend über den Putsch siehe Dreifuss, René Armand, 1964: A Conquista do Estado - Ação Política, Poder e Golpe de Classe, Petrópolis 1981. 
durch die auf konkrete Interessenkonstellationen der Machthaber zurückzuführenden Veränderungen des Verfassungstextes und der Ausnahmegesetze jede rechtlich relevante Herrschaftskontrolle oder jeder Machtwechsel praktisch ausgeschlossen. Es geht hierbei genau besehen um semantische Verfassungen im Sinne Loewensteins oder nach einer von mir vorgeschlagenen Umbenennung um instrumentalistische Verfassungen. Dabei dienen die Verfassungsgesetze nicht der Beschränkung der politischen Macht, sondern sie fungieren als Werkzeug der faktischen Herrscher ${ }^{7}$ so, daß diese personalistisch oder als unpersönliche "Bürokratie" auftreten. ${ }^{8}$ Die Machthaber benutzen die Verfassungstexte bzw. Ausnahmegesetze als reine Mittel der Herrschaftsdurchsetzung, ohne an sie konsequenterweise gebunden zu sein. Die Herrscher verfügen über die ,Werkzeuge“ und können sie ohne jede ernstzunehmende rechtliche Beschränkung umbauen oder ersetzen. ${ }^{9}$

Der militärische Autoritarismus wurde in den sechziger und siebziger Jahren im Rahmen einer konfliktreichen sozialen Situation in Lateinamerika, in der die Umwälzung der gesellschaftlichen Ordnung im Mittelpunkt der politischen Diskussion stand, und unter starkem Einfluß der als "kalter Krieg" bezeichneten ideologischen Spaltung der Welt eingeführt. Die "Wiederherstellung der Ordnung" durch die freilich Konflikte verdrängende und den sozialen status quo beibehaltende Militärherrschaft wurde als einzige politische Alternative von den dominierenden Kräften im Inland und Ausland hingestellt. Aber die konfliktgeladenen Probleme, die in erster Linie auf die gesellschaftliche "Exklusion" großer Teile der Bevölkerung zurückgehen, wurden nicht abgeschwächt. Im Gegenteil hat sich die soziale und ökonomische Lage während der lateinamerikanischen Autokratien der letzten dreißig Jahre in der Regel verschärft. Genaugenommen trugen die Militärregimes, indem sie die Verfassungen als Werkzeuge in Anspruch nahmen, nicht zur "Wiederherstellung der Ordnung" bei, sondern eher zur Gewährleistung von Privilegien und folglich zur Verstärkung von Schichtungsklüften. Damit hing die Verschärfung der sozialen Probleme zusammen, die heftige Resonanz auf der politischen Ebene fand. Mit der Zeit führte die als "im Widerspruch zu den Interessen der Mehrheit" kritisierte, manifest repressive Politik zu Oppositionsbewegungen gegen die Regierung und Widerstände gegen die herrschende soziale Ordnung sowie zur internationalen "Empörung" gegenüber der "Verletzung der Menschenrechte". Unter Zwang von Innen und von Außen verloren die Militärregimes ihre Unter-

Loewenstein, Karl, Verfassungslehre (Fn. 2), S. 153f.; ders., Gedanken über den Wert von Verfassungen in unserem revolutionären Zeitalter, in: Arnold J. Zurcher (Hrsg.), Verfassungen nach dem Zweiten Weltkrieg, dt. Übers.v. Ebba Vockrodt, Meisenheim am Glan 1956, S. 223.

Als Beispiel sind jeweils die chilenische Autokratie, die an die Person des Generals Augusto Pinochet stark gebunden war, und das brasilianische autoritäre Regime, das mit der "Militärbürokratie" so identifiziert wurde, daß der periodische Wechsel des Staatsoberhaupts innerhalb der Armee ohne große Schwierigkeiten und Risiken für die Machtstruktur laufen konnte.

Neves, Marcelo, Verfassung und Positivität des Rechts in der peripheren Moderne: Eine Theoretische Betrachtung und eine Interpretation des Falls Brasilien, Berlin 1992, S. 69 [rezensiert in VRÜ 29 (1996), S. 398, von A. Krell; Anm. d. Red.]. 
stützung, und es setzte sich ein Diskurs für die "Wiederherstellung der Demokratie" durch, der ohne weiteres von den inländischen und ausländischen Kräften, die früher für die "Wiederherstellung der Ordnung" eintraten, übernommen wurde. In diesem Zusammenhang ereignete sich ab den Achtziger Jahren der Übergang zur "Demokratisierung" in den lateinamerikanischen Ländern.

III.

Die sich seit dem letzten Jahrzehnt als herrschende Tendenz abzeichnende "Demokratisierung" der lateinamerikanischen Länder führt auf keinen Fall zum Aufbau, geschweige denn zur Verwirklichung des demokratischen Rechtsstaats nach dem europäischen und nordamerikanischen Vorbild. ${ }^{10}$ Es handelt sich vielmehr um demokratische Verfassungsurkunden, denen es in weitem Umfang an normativer Kraft fehlt.

Hier ist es wichtig, die von der Strukturierenden Rechtslehre vorgeschlagene Unterscheidung von Rechtstext und Rechtsnorm anzuführen ${ }^{11}$. Im Rahmen dieses theoretischen Paradigmas läßt sich feststellen, daß nicht nur die "Entscheidungsnorm" (die individuelle Norm), sondern auch die "Rechtsnorm" (die allgemeine Norm) im Konkretisierungsprozeß erzeugt wird. Der Verfassungstext stellt sich einerseits als "das wichtigste Eingangsdatum" dieses Prozesses dar. ${ }^{12}$ Andererseits muß die konkretisierende Entscheidung dem Text

Es wird dann von "einer anderen Institutionalisierung" gesprochen (O'Donnell, Guillermo, Uma outra institucionalização, in: Lua Nova - Revista de Cultura e Política [São Paulo: CEDEC], N. 37, 1996 S. 5-31. Umfassend über die "Grenzen der demokratischen Konsolidierung in Lateinamerika" siehe u. a. Alcántara, Manuel u. Crespo, Ismael (Hrsg.), Los Límites de la Consolidación Democrática en América Latina, Salamanca 1995.

Vgl. Müller, Friedrich, Juristische Methodik, 6. Aufl., Berlin 1996, S. 122ss.; ders., Strukturierende Rechtslehre, 2. Aufl., Berlin 1994, insbes. S. 147-67 u. 234-40; ders., Die Positivität der Grundrechte: Fragen einer praktischen Grundrechtsdogmatik, 2. Aufl., Berlin 1990, S. 126ss.; ders., Essais zur Theorie von Recht und Verfassung, hrsg. v. Ralph Christensen, Berlin 1990, insbes. S. 20; Christensen, Ralph, Der Richter als Mund des sprechenden Textes. Zur Kritik des gesetzespositivistischen Textmodells, in: Friedrich Müller (Hrsg.), Untersuchungen zur Rechtslinguistik, Berlin 1989, S. 47-91; S. 78ss.; Jeand'Heur, Bernd, Gemeinsame Probleme der Sprachund Rechtswissenschaft aus der Sicht der Strukturierenden Rechtslehre, in: ibid., S. 17-26, insbes. S. 22f. Trotz anderer theoretischer Voraussetzungen siehe ähnlich Grau, Eros, La Doppia Destrutturazione del Diritto: una teoria brasiliana sull'interpretazione, ital. Übers. v. E. Albesano, Milano 1996, S. 55 ff.

Müller, Friedrich, Essais zur Theorie von Recht und Verfassung (Fn. 11), S. 20; vgl. ibid., S. 127 u. 129; Jeand'Heur, Bernd, Gemeinsame Probleme der Sprach- und Rechtswissenschaft... (Fn. 11), S. 22. 
"zurechenbar" sein, obgleich sie die unterschiedlichsten Inhalte aufweisen kann. ${ }^{13}$ Aus rechtsstaatlichen Gründen wird "der Wortlaut als Begrenzung des Spielraums zulässiger Konkretisierung" konzipiert. ${ }^{14}$ Im Fall der Demokratisierung der lateinamerikanischen Staaten aber ist es eindeutig, daß die nach dem demokratisch-rechtsstaatlichen Muster aufgebauten und in Kraft gesetzten Verfassungstexte im Verlauf des Konkretisierungprozesses unter einer umfassenden semantischen Entstellung leiden, besonders was die Erklärung der (individuellen, sozialen und kollektiven) Grundrechte, die Gewaltenteilung und die demokratischen Wahlverfahren angeht. Ich habe im Hinblick auf die brasilianische Verfassung von entkonstitutionalisierender Konkretisierung der Verfassungstexte gesprochen. ${ }^{15}$ Dabei gibt es kein konsistentes normatives Verhältnis zwischen dem Text und den konkretisierenden Tätigkeiten. Die Verfassungstexte sind eine entfernte Bezugsgröße nicht nur für die Privatperson im allgemeinen, sondern in erster Linie für die staatlichen Akteure, deren Praxis sich häufig am Rand des vertexteten Verfassungsmodells abspielt. Und dies tritt sowohl in den rechtswidrigen Gewalttätigkeiten des Polizeiapparats und in der verbreiteten Korruption in der Verwaltung ebenso zutage wie auch in den gesetzwidrigen Tauschbeziehungen und Kampagnenfinanzierungen im Wahlverfahren, in den korrupten Verhandlungen im Parlament und nicht zuletzt in der Unterordnung der Gerichtsbarkeit unter die politischen und wirtschaftlichen Kriterien, unter die guten Beziehungen usw. ${ }^{16}$ Anders als bei den autokratischen Erfahrungen handelt es sich also bei der Demokratisierung lateinamerikanischer Länder nicht um die direkte Negation des Rechtsstaats auf der Ebene der Verfassungstextgebung bzw. Ausnahmegesetzgebung, sondern um im Lauf des Konkretisierungsvorgangs auftretende Hindernisse seiner Konstruktion bzw. Verwirklichung, die zur normativen Verzerrung des mit rechtsstaatlichem Inhalt verkündeten Verfassungstextes führen.

Das semantische Problem der normativen Verzerrung des Verfassungstexts hängt mit pragmatischen Bedingungen des Konkretisierungsprozesses zusammen. Im Hinblick auf den demokratischen Rechtsstaat ist die ganze Öffentlichkeit, sind mit Häberles Worten "alle Staatsorgane, alle öffentliche Potenzen, alle Bürger und Gruppen", in diesen Prozeß

Müller, Friedrich, Juristische Methodik - ein Gespräch im Umkreis der Rechtstheorie, ein Interview mit Jan Möller aus Anlaß des Erscheinens der 5. Auflage des gleichnamigen Werkes von F. Müller, in: Verwaltungsrundschau 4, 1994, S. 133-136, S. 134.

Neves, Marcelo, Symbolische Konstitutionalisierung und faktische Entkonstitutionalisierung: Wechsel von bzw. Änderung in Verfassungstexten und Fortbestand der realen Machtverhältnisse, in: Verfassung und Recht in Übersee, 29. Jahrgang - 3. Quartal 1996, S. 309-323, S. 312 ff.

In diesem Kontext ist die Behauptung nicht angemessen: "Ein Gericht ist neutral, desinteressiert, sachorientiert und allein dem Recht verpflichtet - wenn es denn überhaupt irdische Gerechtigkeit gibt, dann ist sie wohl hier zu finden" (Preuß, Ulrich K., Einleitung: Der Begriff der Verfassung und ihre Beziehung zur Politik, in: ders., Zum Begriff der Verfassung: Die Ordnung des Politischen, Frankfurt am Main 1994, S. 7-33, S. 7). 
eingebunden. ${ }^{17}$ Dementsprechend hängt "die normative Kraft der Verfassung" ${ }^{18}$ von der Einschaltung der pluralistischen Öffentlichkeit in den Prozeß der Verfassungskonkretisierung ab. In diesem Sinne stellt sich die Verfassung nach der markanten Formulierung Häberles als "Spiegel von Öffentlichkeit" dar. ${ }^{19}$ Was die Demokratisierung der lateinamerikanischen Länder jedoch charakterisiert, ist genau das Nichtvorhandensein einer pluralistischen Öffentlichkeit, die aus Bürgern bestehen würde, die vom rechtlichen Standpunkt aus egalitär in die Gesellschaft integrierte Individuen wären. Die Privilegien von einer Minderheit und die "Exklusion" der Mehrheit wirken als entscheidende Faktoren der Torpedierung der Verfassung als Grundordnung der politischen und rechtlichen Kommunikation bzw. als rechtspolitisches Modell der Öffentlichkeit. In diesem Kontext muß der Verweis auf die Öffentlichkeit durch die Feststellung spezifiziert werden, daß es sich um eine auf bestimmte Gruppen und Organisationen "eingeschränkte Öffentlichkeit" handelt. Damit hängt unter systemtheoretischem Gesichtspunkt der Mangel an angemessener Selektivität der offiziellen Verfahren der Verfassungsauslegung bzw. -anwendung angesichts der politischen Erwartungen von Individuen und Gruppen zusammen. Nicht nur diese Erwartungen sind weitgehend verfassungsmarginal oder verfassungsdestruktiv; auch die Verfahren führen oft zur "kasuistischen" Verzerrung des normativen Sinnes des Verfassungstextes. Im Rahmen einer destruktiven Praxis gegenüber dessen möglichen normativen Sinngehalten entsprechen ihm weitgehend nicht "kongruent generalisierte normative Verhaltenserwartungen". 20

In ergänzendem Verhältnis zur unzureichenden rechtsnormativen Konkretisierung bzw. Realisierung der Verfassungsurkunden, also zur mangelhaften normativen Kraft der Verfassungen, steht bei der Demokratisierung der lateinamerikanischen Länder das Problem der hypertroph symbolischen Funktion der Verfassunggebung bzw. der Verfassungstexte. ${ }^{21}$ Hier greife ich auf die in der Bundesrepublik relevante Debatte über symbolische Gesetz-

Häberle, Peter, Die offene Gesellschaft der Verfassungsinterpreten: Ein Beitrag zur pluralistischen und ,prozessualen" Verfassungsinterpretation, in: ders., Die Verfassung des Pluralismus: Studien zur Verfassungstheorie der offenen Gesellschaft, Königstein/Ts. 1980, S. 79-105, S. 80, der sich aber auf den Prozeß der Verfassungsinterpretation bezieht. Im Rahmen des Konkretisierungsvorgangs beschränkt sich die Interpretation auf den Text; vgl. z. B. Müller, Friedrich, Juristische Methodik (Fn. 11), S. 272 ff.

Hesse, Konrad, Die normative Kraft der Verfassung, in: ders., Ausgewählte Schriften, hrsg. v. P. Häberle u. A. Hollerbach, Heidelberg 1984, S. 13-18.

Hierzu siehe näher, was vor allem die semantische Präzisierung des Terminus ,symbolisch“ in diesem Kontext angeht, Neves, Marcelo, A Constitucionalização Simbólica, São Paulo 1994. Vgl. auch ders., Verfassung und Positivität des Rechts in der peripheren Moderne (Fn. 9), S. 61-65 u. 104-106; ders., Symbolische Konstitutionalisierung und faktische Entkonstitutionalisierung (Fn. 15), S. 316; Bryde, Brun-Otto, Verfassungsentwicklung: Stabilität und Dynamik im Verfassungsrecht der Bundesrepublik Deutschland, Baden-Baden 1982, S. 27-29. 
gebung zurück. $^{22}$. Während aber die symbolische Gesetzgebung nur spezifische Sektoren des Rechtssystems berührt, trifft die symbolische Konstitutionalisierung infolge der größeren Weite des materialen und personalen Geltungsbereiches des Verfassungsrechts den Kern des Rechtssystems und belastet seine ganze operative Struktur. Hier wird nicht verkannt, daß auch die "normativen Verfassungen" Westeuropas und Nordamerikas eine symbolische Funktion ausüben ${ }^{23}$; ebensowenig wird übersehen, daß die Unterscheidung zwischen "normativer Verfassung" und "symbolischer Verfassung" relativ ist und es sich "eher um zwei Endpunkte auf einer Skala als um eine Dichotomie" handelt. ${ }^{24}$ Die symbolische Funktion der "normativen Verfassungen" ist jedenfalls an ihre rechtlich-instrumentelle Relevanz gekoppelt, d.h. an einen hohen Grad von verallgemeinerter normativer Konkretisierung der Verfassungsbestimmungen.

Im Fall der symbolischen Konstitutionalisierung als Kennzeichen der Demokratisierung Lateinamerikas folgt jedoch auf die Verfassunggebung bzw. Verfassungsreform keine verallgemeinerte rechtliche Normativität, keine umfassende normative Konkretisierung des

Kindermann, Harald, Symbolische Gesetzgebung, in: Dieter Grimm / Werner Maihofer (Hrsg.), Gesetzgebungstheorie und Rechtspolitik (Jahrbuch für Rechtssoziologie und Rechtstheorie 13), Opladen 1988, S. 222-245; ders., Alibigesetzgebung als symbolische Gesetzgebung, in: Rüdiger Voigt (Hrsg.), Symbole der Politik, Politik der Symbole, Opladen 1989, S. 257-273; Bryde, BrunOtto, Effektivität von Recht als Rechtsproblem, Vortrag gehalten vor der Juristischen Gesellschaft zu Berlin am 17. März 1993 (Schriftenreihe der Juristischen Gesellschaft zu Berlin, H. 135), Berlin / New York 1993, S. 12 ff.; Noll, Peter, Symbolische Gesetzgebung, in: Zeitschrift für Schweizerisches Recht (N.F.) 100, 1981, S. 347-364; Hegenbarth, Rainer, Symbolische und instrumentelle Funktionen moderner Gesetze, in: Zeitschrift für Politik 14, 1981, S. 202-204. Diese Debatte geht auf Gusfield, Joseph R., Symbolic Crusade: Status Politics and the American Temperance Movement, Urbana 1963, zurück; vgl. auch ders., Moral Passage: The Symbolic Process in Public Designations of Deviance, in: Social Problems, Vol. 15, N. 2, 1967, S. 175-188; Aubert, Vilhelm, Einige soziale Funktionen der Gesetzgebung, in: Ernst E. Hirsch /Manfred Rehbinder (Hrsg.), Studien und Materialien zur Rechtssoziologie (Sonderheft 11 der Kölner Zeitschrift für Soziologie und Sozialpsychologie), Köln / Opladen 1967, S. 1967, 284-309. Sie soll aber nicht mit derjenigen über symbolische Politik bzw. Recht als Symbolismus verwechselt wird, in deren Rahmen die Politik bzw. das Recht auf die symbolische Dimension zurückgeführt wird. Vgl. jeweils Edelman, Murray, The Symbolic Uses of Politics, Urbana / Chicago / London 1967; ders., Political Language: Words That Succeed and Policies That Fail; New York / San Francisco / London 1977; Arnold, Thurman, The Symbols of Government, New Haven 1935 (5. Aufl. 1948), insbes. S. $33 \mathrm{ff}$.

Hierzu Vgl. z. B. Burdeau, Georges, Zur Auflösung des Verfassungsbegriffs, in: Der Staat 1, 1962, S. 389-404, S. 398; Edelman, Murray, The Symbolic Uses of Politics (Fn. 22), , S. 18 f.; Massing, Otwin, Identität als Mythopoem. Zur politischen Symbolisierungsfunktion verfassungsrechtlicher Spruchweisheiten, in: Riidiger Voigt (Hrsg.), Politik der Symbole, Symbole der Politik, Opladen 1989, S. 235-256; Habermas, Jiirgen, Faktizität und Geltung: Beiträge zur Diskurstheorie des Rechts und des Rechtsstaats, Frankfurt am Main 1992, S. 342.

Bryde, Brun-Otto, Verfassungsentwicklung (Fn. 21), S. 27. 
Verfassungstextes. ${ }^{25}$ Das Unterscheidungsmerkmal ist die Hypertrophie der symbolischen Dimension zu Lasten der rechtlich-instrumentellen Verwirklichung der Verfassungsbestimmungen. Das Problem liegt darin, daß ein Modell vermittelt wird, dessen Verwirklichung nur unter völlig anderen sozialen Bedingungen möglich wäre. Der Verfassungssymbolismus impliziert also eine illusorische Darstellung in Bezug auf die Verfassungswirklichkeit und dient eher der Immunisierung des politischen Systems gegen andere Alternativen. Durch ihn können nicht nur die Probleme und Beziehungen, die auf der Grundlage der jeweiligen Verfassungsbestimmungen normiert würden, unverändert fortdauern, ${ }^{26}$ sondern es kann auch der Weg der sozialen Änderungen in Richtung des proklamierten Verfassungsstaates blockiert werden. Zum Diskurs der Macht gehören also die ständige Berufung auf die Verfassungsurkunde als normative Struktur der Gewährleistung der Grundrechte, der "Gewaltenteilung" und der demokratischen Wahl, und auch der rhetorische Rekurs auf diese Institutionen als Errungenschaften der Regierung bzw. des Staats sowie als Beweise des Vorkommens von Demokratie im Lande. Die ideologisch aufgeladene Formel "demokratische Gesellschaft" wird von den mit "symbolischen Verfassungen" Regierenden so regelmäßig benutzt wie von ihren Kollegen unter "normativen Verfassungen", wobei unterstellt wird, daß es sich um dieselbe Verfassungswirklichkeit handelt. Daraus entspringt eine pragmatische Verzerrung der Verfassungssprache, die zwar einerseits die soziale Spannung vermindert und die Wege zur Veränderung der Gesellschaft verstellt, indem sie das System gegen andere Alternativen immunisiert, andererseits aber in Extremfällen zum Mißtrauen des Publikums gegen das politische System und die staatlichen Akteure führen kann. In dieser Hinsicht hat selbst die ideologische Funktion der symbolischen Konstitutionalisierung ihre Grenzen, so daß sich die Situation paradoxerweise in ihr Gegenteil verkehren kann, im Sinne einer Bewußtmachung des Auseinanderklaffens von politischer Handlung und konstitutionalistischem Diskurs.

Die symbolische Konstitutionalisierung bezieht sich jedoch nicht nur auf die "legitimierende" Rhetorik der Regierungen bzw. Machtinhaber. Auch im politischen Diskurs der Kritiker des Herrschaftssystems spielt die Berufung auf die im Verfassungstext proklamierten Werte eine relevante symbolische Rolle. So ist beispielsweise die politisch-soziale Rhetorik der "Menschenrechte" bzw. der "Staatsbürgerschaft" paradoxerweise umso intensiver, je geringer der Grad der normativen Konkretisierung des Verfassungstextes ist. Es geht bei der symbolischen Konstitutionalisierung dennoch nicht um ein Nullsummenspiel im politischen Kampf um den Aufbau und die Verwirklichung des demokratischen Rechtsstaates, denn im Gegensatz zu den autokratischen Verfassungschartas und Ausnahmegesetzen ermöglicht sie die Entstehung von sozialen Bewegungen und Organisationen, die dafür

Villegas, Maurício García, La Constitución e su Eficacia Simbólica, in: Revista Universidad de Antiorquia, Bd. LX, N. 225 (Medellin), 1991, S. 4-21, S. 20, mit Bezug auf die kolumbianische Erfahrung.

26

Bryde, Brun-Otto, Verfassungsentwicklung (Fn. 21), S. $28 \mathrm{f}$. 
eintreten, und den Aufbau einer pluralistischen Öffentlichkeit, die trotz ihrer Eingeschränktheit in der Lage ist, sich erfolgreich im Rahmen der verfassungsrechtlichen Verfahren zu artikulieren. Es ist allerdings nicht auszuschließen, daß der Zynismus der Eliten und die Apathie des Publikums ${ }^{27}$ gegenüber dem Erstarren von gravierenden sozialen Problemen zur wirksamen Wiederbelebung des Diskurses für die "Wiederherstellung der Ordnung" führen könnte.

\section{IV.}

Dem oben skizzierten Bild von Verfassungsinstrumentalismus und Verfassungssymbolismus entsprechend beziehen sich die Hindernisse für den Aufbau und Realisierung des demokratischen Rechtsstaates in Lateinamerika nicht nur auf den Autokratismus, sondern auch komplexererweise auf die sogenannte "Demokratisierung". Man stößt gleichzeitig auf die Probleme der Grenzen systemischer Autolegitimation und diskursiver Heterolegitimation des Staates.

Wie ich schon früher am Beispiel Brasiliens festgestellt habe, ${ }^{28}$ ist Luhmanns Auffassung der Autopoiesis auf die Rechtswirklichkeit der peripheren Moderne nicht übertragbar. Die partikularistischen Überlagerungen der Rechtsfragen durch den politischen und den wirtschaftlichen Code machen die Konstruktion der systemischen Autonomie des Rechts unmöglich. Anstelle von Autopoiesis ließe sich dann von Allopoiesis des Rechts spre chen $^{29}$. Das bedeutet, da $\beta$ sich kein "Rechtsfeld" bildet, das fähig dazu wäre, nach seinen eigenen Kriterien und in kongruent generalisierter Weise die von seinen politischen und ökonomischen Kontexten herkommenden Einflüsse "umzuschalten". Die Verwicklung des Rechtscodes mit anderen sozialen Präferenz-Codes wirkt selbst- und fremddestruktiv auf die Systemintegration. Das systemische Problem besteht primär nicht in dem Mangel an kognitiver Offenheit (Fremdreferenz), sondern in der unzureichenden operationellen Geschlossenheit (Selbstreferenz), was die Konstruktion der eigenen Identität des Rechtssystems verhindert. Obwohl solche Identität sich eventuell auf der Ebene der normativen Textstrukturen feststellen läßt, wird sie im Laufe des rechtlichen Konkretisierungsprozesses allmählich zerstört bzw. entstellt. Eben deshalb fehlt es in weiterem Maße an kongruenter

So Kindermann, Harald, Alibigesetzgebung (Fn. 22), S. 270, spezifisch in Bezug auf die Extremfälle von Alibigesetzgebung. 
Generalisierung normativer Verhaltenserwartungen ${ }^{30}$ auf der Basis der Gesetzes- und Verfassungstexte. Daraus ergibt sich, daß die Differenz Recht/Unrecht selbst gesellschaftlich vernebelt wird, sei es mangels Institutionalisierung (Konsens) oder mangels Sinnidentifikation. $^{31}$. Dabei ist die gravierendste Folge die destruktive Unsicherheit in den auf Interessenkonflikten beruhenden Beziehungen.

Was spezifisch die Verfassung angeht, kann man beobachten, daß sie weder als Mechanismus der operativen Geschlossenheit des Rechtssytems noch als strukturelle Kopplung von Politik und Recht ${ }^{32}$ ausreichend funktioniert. Einerseits wird das Verfassungsrecht durch verschiedene gesellschaftliche Faktoren, Kriterien und Präferenz-Codes blockiert, ohne ihre Filterungsfunktion gegenüber den umweltlichen Einflüssen auf das Rechtssystem zureichend zu erfüllen. Andererseits impliziert die selbst- und fremddestruktive Beziehung zwischen Politik und Recht die Implosion der Verfassung als struktureller Kopplung von beiden Systemen, besonders zu Lasten der Autonomie des Rechtssystems. Der Einfluß der Politik auf das Recht wird in großem Ausmaß und relevanterweise nicht gefiltert durch die rechtsstaatlichen Verfassungsverfahren.

Das Problem der Systemautonomie bzw. Autolegitimation des demokratischen Rechtsstaates ist bei allen Divergenzen von Ausgangspunkten von der Frage seiner diskursiven Legitimation in einer pluralistischen, heterogenen Öffentlichkeit untrennbar. Die Auflösung der "präkonventionellen" und "konventionellen" Moralvorstellungen hat in Lateinamerika auf keinen Fall die Konstruktion und Entwicklung des "postkonventionellen Moralbewußtseins" im Sinne Habermas' hervorgebracht, geschweige denn hat sie zum Vorherrschen einer Verfahrensrationalität im öffentlichen Raum geführt. Deswegen findet die moralische bzw. ethische Grundlage der Unparteilichkeit des Rechtsstaats bzw. der Unverfügbarkeit des Rechts als Gegengewicht zu dessen "systemischer Instrumentalisierung" ${ }^{33}$ keinen Platz in den intersubjektiven Rechtsbeziehungen. Die Tendenz ist dann die politische Instrumentalisierung des Rechts, sei es mittels kasuistischer Veränderungen der normativen Strukturen, besonders während der autoritären Perioden, sei es durch das Spiel der parti-

Ich beziehe mich hier auf Luhmanns Definition des Rechts: "... Struktur eines sozialen Systems, die auf kongruenter Generalisierung normativer Verhaltenserwartungen beruht" (Luhmann, Niklas Rechtssoziologie, 3. Aufl., Opladen 1987, S. 105); oder einfach: die "kongruent generalisierten normativen Verhaltenserwartungen" (ibid., S. 99). Mit anderer Formulierung behauptet er, daß "das Recht umfassende Funktionen der Generalisierung und Stabilisierung von Verhaltenserwartungen [erfüllt]" (ders., Rechtssystem und Rechtsdogmatik, Stuttgart 1974, S. 24). Vgl. auch ders., Das Recht der Gesellschaft, Frankfurt am Main 1993, 131 ff.

Über Institutionalisierung, Sinnidentifikation und Normierung als Generalisierungsmechanismen des Rechts, siehe Luhmann, Niklas, Rechtssoziologie, 3. Aufl., Opladen 1987a, S. 94 ff. Hierzu Luhmann, Niklas, Verfassung als evolutionäre Errungenschaft, in: Rechtshistorisches Journal 9, S. 176-220. 
kularistischen Interessen, ${ }^{34}$ die den rechtsnormativen Konkretisierungsprozeß entstellen oder blockieren. In diesem Kontext werden die Menschenrechte und das Prinzip der Volkssouveränität, die nach Habermas "nicht zufällig die Ideen [bilden], in deren Licht das moderne Recht nur noch gerechtfertigt werden kann", ${ }^{35}$ trotz ihrer Erklärung im Verfassungstext durch die Mechanismen der politischen Verzerrung bzw. Zerstörung des Prozesses der Verfassungskonkretisierung eingeschränkt. Damit hängt das Erstarren der Beziehungen von Sub- und Überintegration in die Politik und das Recht zusammen.

Eines der gravierenden Hindernisse für die Konstruktion und die Verwirklichung des Rechtsstaats in Lateinamerika ist die Verallgemeinerung der Beziehungen von Sub- und Überintegration. ${ }^{36}$ Dabei fehlt es an rechtlicher "Inklusion" als Einbeziehung der Gesamtbevölkerung in die Leistungen des positiven Rechts, d.h. Zugang zu diesen Leistungen und damit gleichzeitig Abhängigkeit von ihnen. ${ }^{37}$

34

Was die Rolle der Partikularismen im politischen Spiel angeht, namentlich im Hinblick auf die Demokratisierung lateinamerikanischer Länder, siehe mit anderen Voraussetzungen und Folgerungen O'Donnell, Guillermo, Uma outra institucionalização (Fn. 10), S. 19 ff.

Habermas, Jürgen, Faktizität und Geltung (Fn. 23), S.. 129.

36

Neves, Marcelo, Entre Subintegração e Sobreintegração: A Cidadania Inexistente, in: Dados Revista de Ciência Social (Rio de Janeiro: IUPERJ), Bd. 37, N. 2, 1994, S. 253-276; ders., Verfassung und Positivität des Rechts (Fn. 9), S. 78f. u. $94 \mathrm{ff}$.

37 Luhmann, Niklas, Politische Theorie im Wohlfahrtsstaat, München 1981, S. 25 ff. Ausgehend primär von der Abhängigkeit und nicht vom Zugang unterscheidet Luhmann neuerdings anders als ich zwischen Exklusionsbereich als hochintegriert und Inklusionsbereich als weniger integriert (ders., Das Recht der Gesellschaft [Fn. 30], S. 584f.; ders., Die Gesellschaft der Gesellschaft, Frankfurt am Main, Teilbd. II, S. 631 ff.; ders., Inklusion und Exklusion [Fn. 4,], S. 259f.). Aber nach meiner Formulierung implizieren Subintegration und Überintegrtion jeweils unzureichende "Inklusion", sei es mangels Zugangs zu den systemischen Leistungen, sei es mangels Abhängigkeit von ihnen. Außerdem besteht Luhmann anders als ich darauf, daß trotz der verallgemeinerten Bedingungen von "Inklusion/Exklusion" wie im Fall Lateinamerika die Ausschaltung der Autopoiesis des Rechts nicht vorkommt (ders., Die Gesellschaft der Gesellschaft, S. 632). Aber vorsichtig gibt er zu: "Diese Differenz von Inklusion und Exklusion hat gravierende Effekte, weil sie einerseits durch die funktionale Differenzierung der Weltgesellschaft ausgelöst ist, andererseits die regionale Herstellung der Bedingungen funktionaler Differenzierung behindert, wenn nicht verhindert (ders., Die Gesellschaft der Gesellschaft, Teilbd. I, S. 169 - Hervorhebung von mir). Dabei wird dennoch die Variable Inklusion/Exklusion zu einer Metadifferenz bzw. Metacode, der die Codes aller Funktionssysteme mediatisiert" (ders., Die Gesellschaft der Gesellschaft, S. 632; ders., Das Recht der Gesellschaft, S. 583). Aber wenn dem so ist, scheint es mir sehr umstritten, weiter zu behaupten, daß die moderne Gesellschaft durch einen Primat funktionaler Differenzierung charakterisiert ist und daß die Differenz von System und Umwelt innergesellschaftlich die Hauptdifferenz ist. Um mit der Behauptung konsequent zu sein, daß die Differenz von Inklusion und Exkusion" als ein die anderen Codes mediatisierender Metacode dient, müßte man m. E. daraus die Folge ziehen, daß die Weltgesellschaft primär nach dieser Metadifferenz differenziert ist. 
Was die Subintegrierten anbelangt, verallgemeinern sich die konkreten Beziehungen, in denen sie keinen Zugang zu den Leistungen des Rechtssystems haben, obgleich sie abhängig von ihnen bleiben. Die "Sub-Bürger" sind also nicht exkludiert. Obwohl ihnen die wirklichen Bedingungen für die Ausübung der in der Verfassung erklärten Grundrechte fehlen, sind sie nicht von den Verpflichtungen und Verantwortungen befreit, die ihnen die staatliche Zwangsordnung auferlegt. Eher bleiben sie der Bestrafungsstruktur des "Staatsapparates" streng unterworfen. Jedenfalls spielen die Grundrechte keine relevante Rolle im Horizont des Bewußtseins und Handelns der Subintegrierten. Für diese haben die Verfassungsvorschriften nur in deren freiheitsbeschränkenden Wirkungen Relevanz. Das gilt für das Rechtssystem im Ganzen: Die in verschiedenen Graden und Hinsichten "marginalisierten" Unterschichten (d.h. der größte Teil der Bevölkerung) werden als Verpflichtete, Angeklagte usw. in das System integriert, nicht als Berechtigte, Kläger usw. Im Bereich der Verfassung nimmt das Problem der Subintegration aber eine spezielle Tragweite in dem $\mathrm{Maße}$ an, wie in Bezug auf die Angehörigen der Unterschichten die Verletzungen der Grundrechte besonders im Rahmen der repressiven Tätigkeit des "Staatsapparates" durchgeführt werden.

Die Subintegration der Massen ist untrennbar von der Überintegration der privilegierten Gruppen, die vor allem in Gestalt bzw. mit Hilfe der "Staatsklasse/Staatsbürokratie" ihre verfassungsaushöhlenden und -durchbrechenden Handlungen ${ }^{38}$ ausführen. Zwar benutzen sie - soweit es prinzipiell zugunsten ihrer Interessen ist bzw. zum Schutz der "sozialen Ordnung" - regelmäßig den demokratischen Verfassungstext; tendenziell aber, insofern die Verfassung ihrem politischen und ökonomischen Handlungsspielraum bedeutende Grenzen setzt, wird sie außer acht gelassen. Die Verfassung fungiert dann nicht als Horizont des rechtspolitischen Handelns und Erlebens der Machthaber, sondern als ein je nach der konkreten Interessenkonstellation von ihnen zu gebrauchendes, zu mißbrauchendes oder nicht zu gebrauchendes Angebot.

Mit dem Problem der Subintegration und Überintegration hängen in der lateinamerikanischen Rechts- und politischen Wirklichkeit die paradoxen komplementären Verhältnisse zwischen Legalismus und Straflosigkeit zusammen. Der Legalismus bedeutet in diesem Kontext keineswegs das Vorhandensein eines von rechtlich gleichen Rechtspersonen bzw. Bürgern konstituierten öffentlichen Raums der Legalität, soll also nicht mit der strengen Durchsetzung des Legalitätsprinzips verwechselt werden. Er impliziert eher einen einseitigen Gesetzesfetischismus, der als Mechanismus der sozialen Diskriminierung funktioniert.

Ich greife hier auf Grimms Unterscheidung zwischen einerseits der "verfassungsausfüllenden Verfassungswirklichkeit" und andererseits der "verfassungsaushöhlenden" und der "verfassungsdurchbrechenden Verfassungswirklichkeit" zurück (Grimm, Dieter, Verfassung, in: Staatslexikon: Recht · Wirtschaft · Gesellschaft, hrsg. v. der Görres-Gesellschaft, 7. Aufl., 5. Bd., Freiburg / Basel / Wien 1989, Sp. 633-643, Sp. 637). 
Die legalistische Interpretation wird normalerweise angewandt auf diejenigen, die nicht in der Lage sind, ihre eigenen Rechte wahrzunehmen, selbst wenn diese gesetzlich und verfassungsmäßig "gewährleistet" sind. Es handelt sich um fehlenden Zugang zum Recht. Die entsprechenden Personen und sozialen Gruppen werden in der Regel nur dann durch den einseitigen Legalismus beachtet, wenn sie zu ihren Ungunsten, also als Schuldige, Angeklagte, Sträflinge usw., mit dem System in Kontakt kommen, aber nicht als Rechtsinhaber. Hier ist es sinnvoll, von Subintegration in das Rechtssystem zu sprechen: Die Einzelwesen sind den Zwangsvorschriften streng untergeordnet, haben aber keinen Zugang zu den Rechten. Als parteiisch und diskriminierend widerspricht die gesetzestreue Strenge der eigenen Legalität, die eine egalitäre Verallgemeinerung der Inhalte und Verfahren der Rechtsordnung impliziert.

Anderseits ist das Verhältnis zwischen Recht und Gesellschaft in Lateinamerika durch Straflosigkeit gekennzeichnet. Häufig stellt sich heraus, da $\beta$ den Rechtswidrigkeiten verschiedenster Art, vor allem im Bereich der Kriminalität, nicht die gesetzlichen Strafmaßnahmen folgen. Man könnte behaupten, daß die systematische Straflosigkeit im Gegensatz zum Legalismus steht. Dieser Gegensatz erweist sich aber in einer sorgfältigen Analyse als scheinbarer. Während die legalistische Strenge sich zuerst gegen die Subintegrierten wendet, hängt die Straflosigkeit in der Regel mit der Welt der Privilegien der rechtlich Überintegrierten zusammen. Eine Person bzw. eine Gruppe läßt sich als überintegriert im Hinblick auf das Recht definieren, sofern diese als Inhaber von Rechten und rechtlich erteilten Befugnissen auftritt, ohne sich zugleich den Verpflichtungs- und Verantwortlichkeitsvorschriften zu unterstellen. Es ist ganz klar, da $\beta$ es den "durchweg Überintegrierten" (sowie den "absolut Subintegrierten") nicht gibt. Aber im lateinamerikanischen Zusammenhang ist das Vorhandensein von Personen notorisch, die sich am privilegierten Pol der Überintegrations- und Subintegrationsverhältnisse befinden. Deswegen können sie in ihrer Erwartungsorientierung und Handlungsweise mit einer weitgehenden Straflosigkeitswahrscheinlichkeit rechnen.

Die Privilegien der Straflosigkeit implizieren den Bruch der Autonomie/Identität der Rechtssphäre durch politische, wirtschaftliche und auf "gute Beziehungen" gerichtete Blockierungen. Man bleibt straflos, weil man mehr politische oder ökonomische Macht oder einfach "bessere Beziehungen" zu den zuständigen Beamten bzw. Machthabern hat. In diesem Zusammenhang werden die nach den Verfassungs- und Gesetzestexten verallgemeinerten Kriterien der spezifisch rechtlichen Bewertung hintangesetzt, hauptsächlich zugunsten der Vorherrschaft der systemischen Macht- und Geldkriterien.

Der Sachverhalt rechtlicher, sozial vermittelter Straflosigkeit trifft systematisch bestimmte Gruppen der Gesellschaft und stellt eine skandalöse Diskriminierung ihnen gegenüber dar (das ist z. B. der Fall bei den wiederholten Massakern an sog. "Straßenkindern", "landlosen 
Bauern" und Indianern) ${ }^{39}$ Umfassend läßt sich behaupten: Während die Opfer der unbestraften Verbrechen in der Regel die sozial Schwächeren sind, sind die Rechtsbrecher bzw. die dafür Verantwortlichen privilegierte Personen und Gruppen, oder diejenigen, die mit ihnen direkt oder indirekt durch Interessen verbunden sind.

V.

Die Verbindung von Legalismus und Straflosigkeit in Lateinamerika, die auf die Verallgemeinerung der Beziehungen von Subintegration und Überintegration zurückgeht, verhindert die Strukturierung der Öffentlichkeit als Legalitätsraum bzw. die Konstruktion einer verfassungsmäßigen Ordnung und folglich die Verwirklichung des demokratischen Rechtsstaats. Dieser erfordert die Konkretisierung demokratischer, verfassungsrechtlich vorgesehener Verfahren, die eine politisch-rechtlich symmetrische Integration von Bürgern und Gruppen in die entsprechende Gesellschaft ermöglicht. Dafür ist es nicht hinreichend, daß die typischen Verfahren des demokratischen Rechtsstaats in den Verfassungs- und Gesetzestexten vorgesehen sind - das Gerichtsverfahren, das auf dem due process of law beruht, das gesetzgebende bzw. parlamentarische Verfahren, das durch die freie Diskussion zwischen Regierung und Opposition aufgebaut wird, und das Wahlverfahren, das die verschiedensten politischen Kräfte im Streit um die Staatsmacht mobilisieren kann. Es ist unerläßlich, da $\beta$ diese Verfahren im Verlauf der Verfassungskonkretisierung nicht straflos verzerrt werden. Das erfordert soziale Bedingungen, die allgemein die Identifizierung der demokratisch-rechtsstaatlichen Verfahren und einen umfassenden Konsens über dieselben möglich machen.

Selbstverständlich kann die Problemlösung nicht auf die Veränderung des Verfassungstextes zurückgeführt werden. Vielmehr ist zu vermuten, daß die verfassungsreformistische Rhetorik, die in diesem Jahrzehnt besonders in Brasilien sehr stark auftritt, in erster Linie eine symbolische Rolle spielt. Regierungsprogramme werden auf Verfassungsreformprogramme reduziert, diese ihrerseits werden nicht selten durchgeführt, dennoch bleiben die sozialen Strukturen und Machtverhältnisse im Wesentlichen unberührt. Die "Entkonstitutionalisierung" gilt z. B. als Allerheilmittel im Diskurs der Macht, sozusagen als Zauberlösung für alle Verfassungsprobleme. Man greift hier unkritisch auf die in den letzten Jahren hierzulande sehr lebhafte Debatte um Verrechtlichung zurück. Dabei wird nicht nur

39

Unter diesen Umständen ist es nicht angebracht, nach der "republikanischen" Verfassungskonzeption von "constant reach for inclusion of the other, of the hitherto excluded" (Michelman, Frank, Law's Republic, in: Yale Law Journal, Vol. 97, N. 8, July 1988, S. 1493-1537, S. 1529; vgl. Habermas, Jürgen, Faktizität und Geltung [Fn. 23], S. 334) zu sprechen, geschweige denn hat die normative Vorstellung von "Differenzempfindlicher Inklusion" (Habermas, Jürgen, Die Einbeziehung des Anderen: Studien zur politischen Theorie, Frankfurt am Main 1996, S. 172 175) Kontextreferenz. 
verkannt, daß die Konkretisierung von früheren synthetischen Verfassungen noch problematischer war, sondern vor allem auch, daß das Problem in Lateinamerika eher in der entrechtlichenden politischen Wirklichkeit und in der entkonstitutionalisierenden Rechtswirklichkeit liegt. Dessen Lösung läuft also nicht auf eine angebliche Entkonstitutionlisierung bzw. Entrechtlichung auf der Ebene des Verfassungstextes hinaus, sondern sie hängt zunächst mit der Konstitutionalisierung der Rechtswirklichkeit und mit der Verrechtlichung der politischen Verhältnisse zusammen. Zwar geht das Problem weit über die Politik und das Recht hinaus, es ist auf umfassende soziale Voraussetzungen angewiesen und nicht zuletzt von weltgesellschaftlichen Bedingungen abhängig; aber es kann nur mit dem Abbau von Illusionen und sogar dem Entlarven von Manipulationen im Hinblick auf die Rechtspolitik ernsthaft angegangen werden. 
national community of states, their constitutional systems and their position in international law. Other aspects the Journal dealt with over the last decades are the validity of customary norms of international law for newly independent states, weighted voting in international organizations, the shape of a new international economic order, especially the right to development and the acknowledgement of resolutions of international organizations as new sources of international law, to name just as few. The article presents and considers these issues in the context of the overall developments of international law.

\section{The Expansion of the European Union: The Relevant Provisions of European Law}

\section{By Thomas Bruha and Oliver Vogt}

The European Union is facing the most extensive expansion in its history. The European Commission suggested that, in 1998, five central- and eastern European states and Cyprus should be invited to first talks on their accession to the Union. Since five more central- and eastern European states as well as Turkey, Malta and Switzerland have already applied, too, the European Union may amount to up to 35 member states after the next enlargement. Since its Essen summit in 1994, the European Union is following a double strategy in preparation for expansion, a "pre-accession" strategy for the applying states and fundamental institutional reforms for the Union itself.

In view of these developments, the provisions of the Union treaty have been emended on the 1997 Amsterdam Council of Heads of States. The article deals with the conditions of the expansion as laid down in the new Art. O European Union Treaty on the admission of new member states, featuring especially the geographical, historical and political aspects of "Europe", and comparing it to the criteria prepared on the 1993 Copenhagen summit. Emphasizing the forthcoming eastern expansion debate, the article proceeds to survey the present situation of the five candidates in view of the new criteria. It concludes with a glance at a new dimension of the debate: the development of a European "civil society". Much of this is relevant far beyond the limits of the present or future European Union.

\section{Latin American Constitutions: Between Autocracy and Democratization}

\section{By Marcelo Neves}

The constitutional development of the Latin American countries in the past three decades is characterized by a tendency shifting from autocracy in the sixties and seventies to democratization in the eighties, with growing stability in the nineties. The question arises whether 
these new political structures are consolidated and whether the constitutional documents are normative models put to practice in reality. The article does not try to give a comparative overview over the constitutions in Latin America, but tries to show some general trends of constitutional developments on the continent.

The author shows that the instrumentalization of constitutions is an expression of autocracy and constitutional symbolism is an expression of democratization, and he goes back to consider the authoritarian military regimes of the sixties and seventies in this regard. Drawing a line between legal text and legal norm, the author notes that the democratization of the past years has led to insufficient effectiveness and a lack of normative force of constitutional instruments. Characteristic for the relationship between law and society in Latin America is the legalistic and severe punishment against the ordinary, 'subintegrated' people on the one hand and the exemption from punishment for the privileged on the other hand one of the roots of the harsh discrimination of certain groups of the society, such as the street children or the Indians. The author considers this gap between legalistic stringency and exemption from punishment as one fundamental obstacle to the implementation of the norms providing rule of law and democracy. He suggests that the existing processes for the integration of all strata of society into public life should be accompanied by criminal punishment and social conditions allowing the identification of the democratic and due process of law and making a corresponding social consensus possible.

\section{Arabian Constitutions and the Issue of "Islamic" Human Rights}

\section{By Hans-Georg Ebert}

The vast majority of Arabian countries have a written constitution or constitutional documents as a foundation for the organization and structure of the state as well as the relationship to the people. The article deals with the influence of Islamic rules and principles on constitutional law and practice in the field of human rights.

The author first introduces into the specific situation of human rights in the Islamic context, then deals with the influence of Islamic rules and principles on the right to equality before the law, the freedom of religion and the doctrine of sovereignty of the people. He concludes that the provisions of Shari'a do not hinder the implementation of human rights per se and, although the doctrine of the sovereignty of the people is not fully compatible with Shari'a, that there is room for a sovereign parliament and thus compatible structures. 